\title{
Erratum to: Secure IoT framework and 2D architecture for End-To-End security
}

\author{
Jongseok Choi ${ }^{1}$ • Youngjin In $^{1}$. Changjun Park ${ }^{1}$ • \\ Seonhee Seok ${ }^{1}$ - Hwajeong Seo ${ }^{1}$. Howon Kim ${ }^{1}$
}

Published online: 5 December 2016

(C) Springer Science+Business Media New York 2016

\section{Erratum to: J Supercomput DOI 10.1007/s11227-016-1684-0}

The Acknowledgements section is missing in the original article. It is given below.

Acknowledgements This work was supported by the Institute for Information and Communications Technology Promotion (IITP) grant funded by the Korea government (MSIP) (R0101-16-0129, Development of High Performance IoT Device and Open Platform with Intelligent Software).

The online version of the original article can be found under doi:10.1007/s11227-016-1684-0.

Howon Kim

howonkim@pusan.ac.kr

Jongseok Choi

js.choi.85@gmail.com

Youngjin In

cubya0104@gmail.com

Changjun Park

pcj006@gmail.com

Seonhee Seok

seokseonhee@gmail.com

Hwajeong Seo

hwajeong84@gmail.com

1 Pusan National University, 6-Eng.Bldg., Jangjeon 2(i)-dong, Geumjeong-gu, Busan 609-735, Republic of Korea 